\title{
Observation of the New Properties of the Secondary Cosmic Rays Lithium, Berillium and Boron with the Alpha Magnetic Spectrometer on the International Space Station
}

\section{Yi Jia*†}

Massachusetts Institute of Technology

E-mail: jiay@mit.edu

\begin{abstract}
We report on the observation of new properties of secondary cosmic rays $\mathrm{Li}, \mathrm{Be}$, and $\mathrm{B}$ measured in the rigidity (momentum per unit charge) range $1.9 \mathrm{GV}$ to $3.3 \mathrm{TV}$ with a total of 5.4 million nuclei collected by AMS during the first five years of operation aboard the International Space Station. The Li and B fluxes have an identical rigidity dependence above $7 \mathrm{GV}$ and all three fluxes have an identical rigidity dependence above $30 \mathrm{GV}$ with the $\mathrm{Li} / \mathrm{Be}$ flux ratio of $2.0 \pm 0.1$. The three fluxes deviate from a single power law above $200 \mathrm{GV}$ in an identical way. This behavior of secondary cosmic rays has also been observed in the AMS measurement of primary cosmic rays $\mathrm{He}, \mathrm{C}$, and $\mathrm{O}$ but the rigidity dependences of primary cosmic rays and of secondary cosmic rays are distinctly different. In particular, above $200 \mathrm{GV}$, the secondary cosmic rays harden more than the primary cosmic rays.
\end{abstract}

The 39th International Conference on High Energy Physics (ICHEP2018)

4-11 July, 2018

Seoul, Korea

* Speaker.

${ }^{\dagger}$ on behalf of the AMS Collaboration. 
Secondary cosmic rays $(\mathrm{Li}, \mathrm{Be}, \mathrm{B}, \ldots)$ are mainly produced in the collisions of primary cosmic rays $(\mathrm{C}, \mathrm{O}, \ldots, \mathrm{Fe})$. They carry information on the history of their travel, and the properties of the interstellar medium (ISM) [1]. The accurate knowledge of secondary cosmic rays is important for the detailed study of the origin and propagation of cosmic rays.

We report the precise measurement of the lithium, beryllium, and boron fluxes in cosmic rays in the rigidity range from $1.9 \mathrm{GV}$ to $3.3 \mathrm{TV}$. This measurement is based on 1.9 million lithium, 0.9 million beryllium, and 2.6 million boron nuclei collected by AMS during the first 5 years (May 19, 2011 to May 26, 2016) of operation aboard the International Space Station (ISS) [2]. The total error on each of the fluxes is $3-4 \%$ at $100 \mathrm{GV}$.

The tracker has nine layers. For $3 \leq Z \leq 5$ events, the charge resolution is $\Delta Z / Z=4-6 \%$ per layer and $2-3 \%$ for the inner tracker ( $L 2$ to $L 8$ combined). Together, the tracker and the magnet measure the rigidity $R$ of charged cosmic rays. For $3 \leq Z \leq 5$ events, the spatial resolution per layer is $5.3-5.8 \mu \mathrm{m}$ in the bending direction [3] so that the maximum detectable rigidity (MDR) is 3.5-3.7 TV over the $3 \mathrm{~m}$ lever arm from $L 1$ to $L 9$ and $\sim 1.4 \mathrm{TV}$ from $L 1$ to $L 8$.

Four planes of time of flight (TOF) discriminate between upward- and downward-going particles. For $3 \leq Z \leq 5$ events, $\Delta Z / Z$ is $3.5-5 \%$ and the overall velocity $\beta=v / c$ resolution has been measured to be $\Delta \beta / \beta^{2}=0.012-0.015$. $\mathrm{Li}, \mathrm{Be}$, and B nuclei traversing AMS were triggered as described in detail in Ref. [4]. The trigger efficiencies for $3 \leq Z \leq 5$ events were measured to be $>$ $98 \%$ over the entire rigidity range.

Figure 1 shows the lithium, beryllium, and boron fluxes as a function of rigidity with the total errors, the sum in quadrature of statistical and systematic errors. In this and the subsequent figures, the points are placed along the abscissa at $\tilde{R}$ calculated for a flux $\propto R^{-2.7}$ [5]. As seen, the $\mathrm{Li}$ and B fluxes have an identical rigidity dependence above $\sim 7 \mathrm{GV}$ and all three secondary fluxes have an identical rigidity dependence above $\sim 30 \mathrm{GV}$. The different rigidity dependence of the Be flux is most likely due to the significant presence of the radioactive ${ }^{10} \mathrm{Be}$ isotope [6], which has a half life of 1.4 MY. To examine the rigidity dependence of the fluxes, detailed variations of the flux spectral indices with rigidity were obtained in a model independent way. The flux spectral indices $\gamma$ were calculated from

$$
\gamma=d[\log (\Phi)] / d[\log (R)]
$$

over rigidity intervals bounded by 7.09, 12.0, 16.6, 22.8, 41.9, 60.3, 192, and $3300 \mathrm{GV}$. The results are presented in Fig. 2 together with the spectral indices of helium, carbon, and oxygen [7]. As seen, the magnitude and the rigidity dependence of the lithium, beryllium, and boron spectral indices are nearly identical, but distinctly different from the rigidity dependence of helium, carbon, and oxygen. In addition, above $\sim 200 \mathrm{GV}, \mathrm{Li}, \mathrm{Be}$, and $\mathrm{B}$ all harden more than $\mathrm{He}, \mathrm{C}$, and $\mathrm{O}$.

To examine the difference between the rigidity dependence of primary and secondary cosmic rays in detail, the ratios of the lithium, beryllium, and boron fluxes to the carbon and oxygen fluxes were computed. The detailed variations with rigidity of the spectral indices $\Delta$ of each flux ratio were obtained in a model independent way using

$$
\Delta=d\left[\log \left(\Phi_{\mathrm{S}} / \Phi_{\mathrm{P}}\right)\right] / d[\log (R)],
$$

where $\Phi_{\mathrm{S}} / \Phi_{\mathrm{P}}$ are the ratios of the secondary to primary fluxes over rigidity intervals [60.3 - 192] GV and [192 - 3300] GV and shown in Fig 3. Above $200 \mathrm{GV}$ these spectral indices exhibit an average hardening of $0.13 \pm 0.03$. 


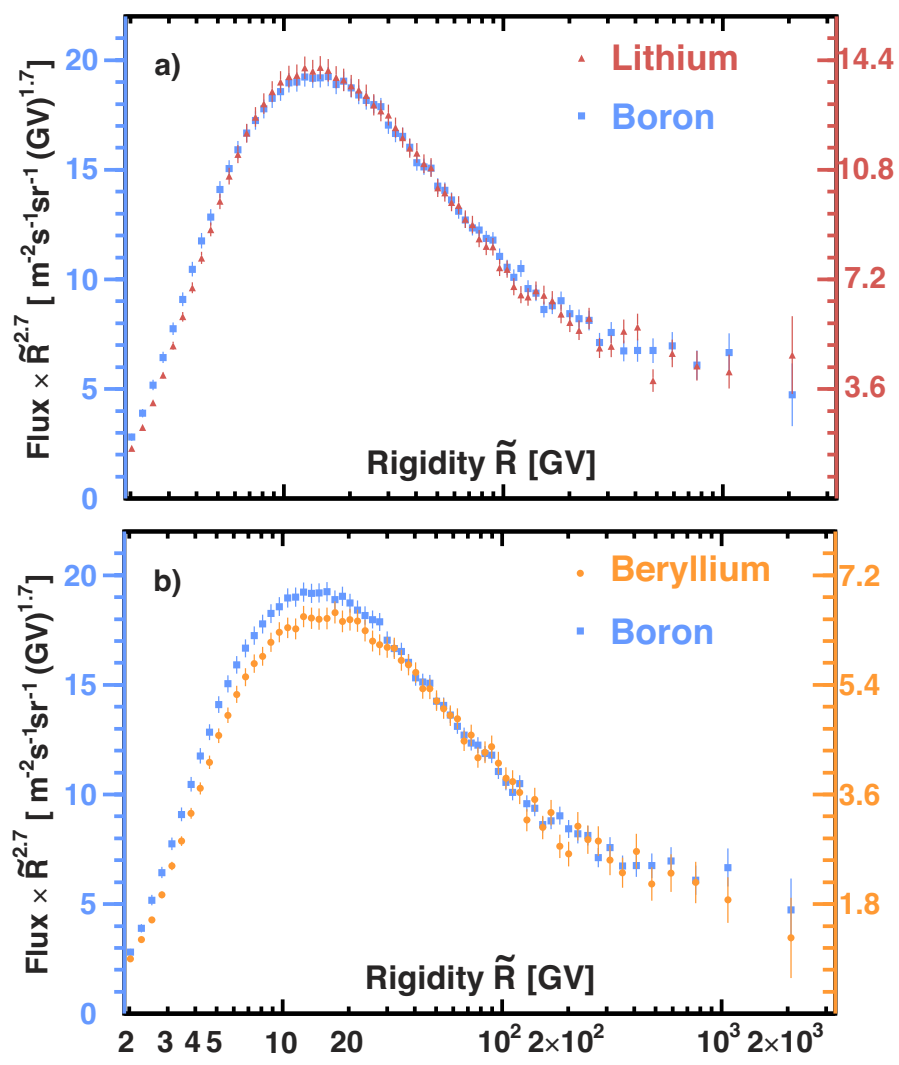

Figure 1: The AMS (a) Li and B and (b) Be and B fluxes multiplied by $\tilde{R}^{2.7}$ with their total errors as a function of rigidity. As seen, the $\mathrm{Li}$ and $\mathrm{B}$ fluxes have identical rigidity dependence above $\sim 7 \mathrm{GV}$ and all three secondary fluxes have identical rigidity dependence above $\sim 30 \mathrm{GV}$.

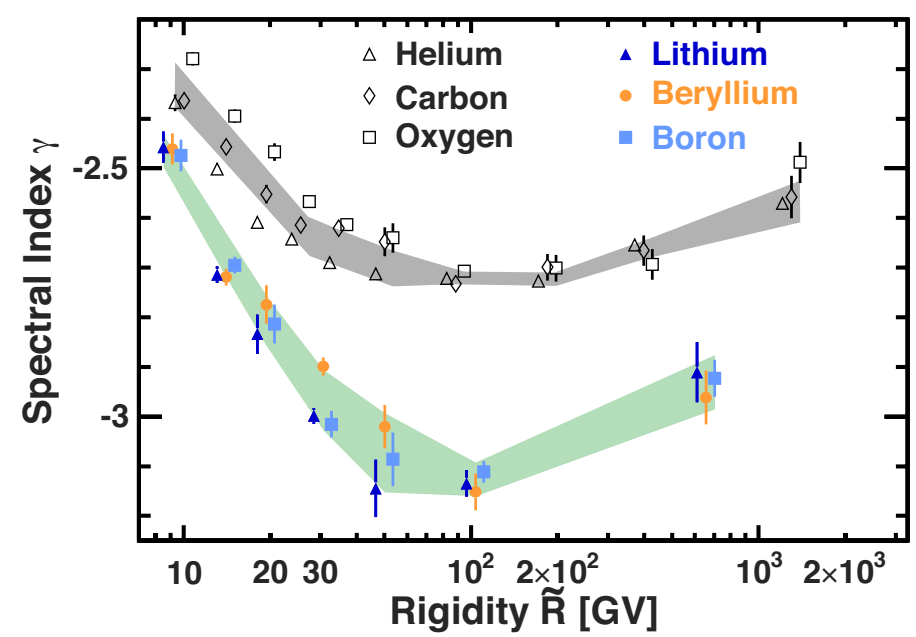

Figure 2: The dependence of the $\mathrm{Li}, \mathrm{Be}$, and $\mathrm{B}$ spectral indices on rigidity together with the rigidity dependence of the $\mathrm{He}, \mathrm{C}$, and $\mathrm{O}$ spectral indices [7]. For clarity, the $\mathrm{Li}, \mathrm{B}, \mathrm{He}$, and $\mathrm{O}$ data points are displaced horizontally. The shaded regions are to guide the eye. As seen, the magnitude and the rigidity dependence of the $\mathrm{Li}, \mathrm{Be}$, and $\mathrm{B}$ spectral indices are nearly identical, but distinctly different from the rigidity dependence of the $\mathrm{He}, \mathrm{C}$, and $\mathrm{O}$ spectral indices. Above $\sim 200 \mathrm{GV}$ the $\mathrm{Li}, \mathrm{Be}$, and B fluxes all harden more than the He, $\mathrm{C}$, and $\mathrm{O}$ fluxes. See also Fig. 3. 


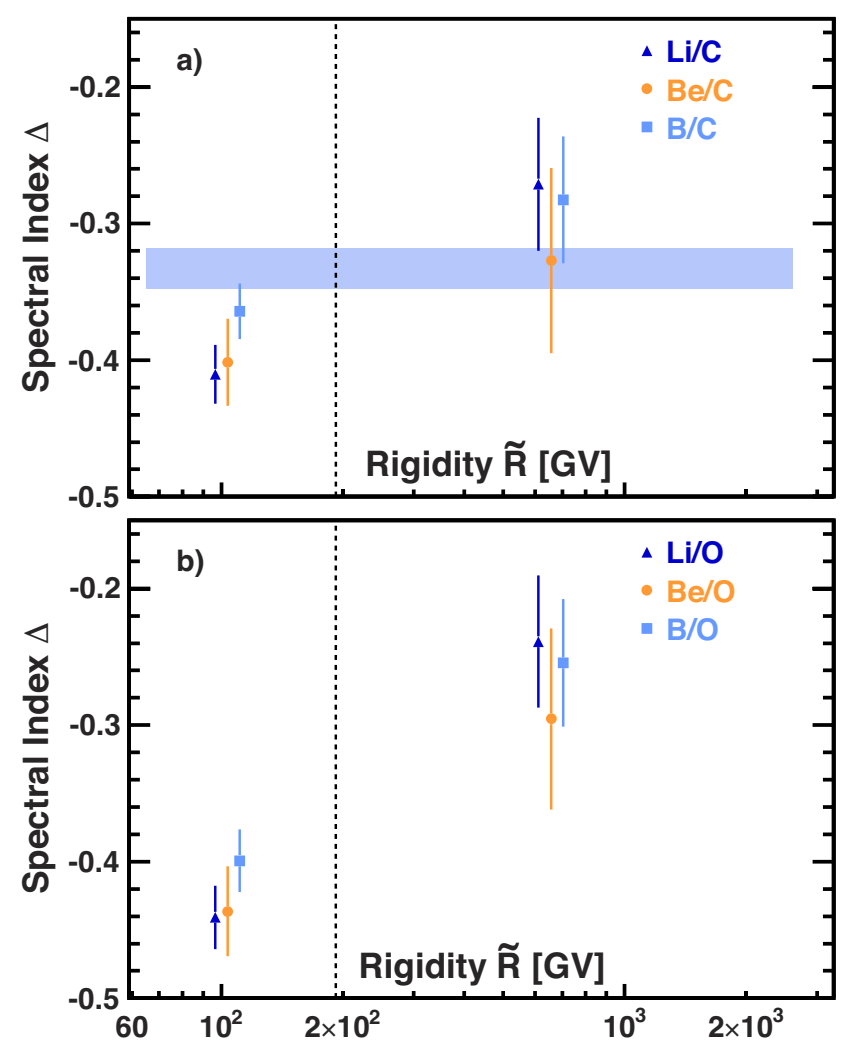

Figure 3: The AMS secondary to primary flux ratio spectral indices $\Delta$ from Eq. (2) as functions of rigidity for (a) $\mathrm{Li} / \mathrm{C}, \mathrm{Be} / \mathrm{C}$, and $\mathrm{B} / \mathrm{C}$. The horizontal band indicates the fit to the $\mathrm{B} / \mathrm{C}$ ratio from our previous publication [8] which is consistent with the results in this Letter. The results for (b) $\mathrm{Li} / \mathrm{O}, \mathrm{Be} / \mathrm{O}$, and $\mathrm{B} / \mathrm{O}$. For (a) and (b) the vertical dashed line shows the interval boundary. On average, the spectral indices of $\mathrm{Li} / \mathrm{C}, \mathrm{Be} / \mathrm{C}$, $\mathrm{B} / \mathrm{C}, \mathrm{Li} / \mathrm{O}, \mathrm{Be} / \mathrm{O}$, and $\mathrm{B} / \mathrm{O}$ above $200 \mathrm{GV}$ exhibit a hardening of $0.13 \pm 0.03$.

\section{References}

[1] I. A. Grenier, J. H. Black, and A. W. Strong, Annu. Rev. Astron. Astrophys. 53, 199 (2015); P. Blasi, Astron. Astrophys. Rev. 21, 70 (2013); A. W. Strong, I. V. Moskalenko, and V. S. Ptuskin, Annu. Rev. Nucl. Part. Sci. 57, 285 (2007); A. Castellina and F. Donato, Astrop. Phys. 24, 1-2 (2005).

[2] M. Aguilar et al., Phys. Rev. Lett. 120021101 (2018).

[3] G. Ambrosi, V. Choutko, C. Delgado, A. Oliva, Q. Yan, and Y. Li, Nucl. Instrum. Methods Phys. Res., Sect. A 869, 29 (2017).

[4] M. Aguilar et al., Phys. Rev. Lett. 115, 211101 (2015).

[5] G. D. Lafferty and T. R. Wyatt, Nucl. Instr. Methods Phys. Res., Sect. A 355, 541 (1995). We have used Eq. (6) with $\tilde{R} \equiv x_{l w}$.

[6] The AMS Collaboration, Measurement of Cosmic Ray Fluxes with the Alpha Magnetic Spectrometer on the International Space Station, (to be published).

[7] M. Aguilar et al., Phys. Rev. Lett. 119, 251101 (2017).

[8] M. Aguilar et al., Phys. Rev. Lett. 117, 231102 (2016). 